\title{
Five Avirulence Genes from Xanthomonas campestris pv. malvacearum Cause Genotype-Specific Cell Death When Expressed Transiently in Cotton
}

\author{
Rob de Feyter, Helen McFadden, and Liz Dennis \\ CSIRO Division of Plant Industry, GPO Box 1600 Canberra, ACT2601, Australia \\ Accepted 3 April 1998.
}

\begin{abstract}
The coding regions from five avirulence (avr) genes from Xanthomonas campestris pv. malvacearum (Xcm), the causal agent of bacterial blight of cotton, were joined to the cauliflower mosaic virus $35 \mathrm{~S}$ promoter and expressed transiently in cotton leaves after Agrobacterium tumefaciens-mediated gene transfer. A genotype-specific necrosis was observed 3 to 6 days post inoculation for constructs derived from avrB4, avrb7, and avrBIn on cotton lines containing the resistance $(R)$ genes $B 4, b 7$, and $B I n$, respectively, but not on susceptible cotton plants. No necrosis was obtained with plasmids that direct expression of avr genes within $A$. tumefaciens, showing that the cotton response required transfer of the genes into the plant cells. Addition of a signal peptide sequence into the avr constructs to target expressed Avr protein to the apoplast significantly reduced the responses. The results indicate that intracellular expression of $\mathrm{Xcm}$ Avr proteins in cotton having the corresponding $R$ gene causes specific host cell death.
\end{abstract}

Additional keywords: hypersensitive responses, transient expression.

As with many interactions between plants and their pathogens, the response of cotton to the bacterial blight pathogen Xanthomonas campestris pv. malvacearum $(\mathrm{Xcm})$ is determined by the presence or absence of specific resistance $(R)$ genes in the plant and particular avirulence (avr) genes in the pathogen. In recent years, numerous $R$ genes have been cloned from plants and many have been shown to be related structurally, having one or more functional domains including nucleotide binding motifs and leucine rich repeat (LRR) regions (Bent 1996). The LRR motifs are thought to be involved in protein-protein interactions. Although many avr genes have been cloned from bacterial pathogens and a few from fungi (reviewed by Keen 1990; Leach and White 1996), it is only recently that their mode of action has begun to be elucidated. In an important advance, Gopalan et al. (1996) showed that the gene product of the Pseudomonas syringae pv. glycinea $a v r B$ gene was delivered by the bacterium, via the Hrp (type

Corresponding author: R. de Feyter

E-mail: Robert.Defeyter@pican.pi.csiro.au
III) secretion system, into soybean cells, and that it induced specific host cell death when the plant cells expressed the corresponding $R$ gene. Similar results have been obtained for avrPto from $P$. syringae pv. tomato in tobacco (Scofield et al. 1996; Tang et al. 1996) and avrBs3 from Xanthomonas campestris pv. vesicatoria in pepper (Van den Ackerveken et al. 1996). These results were extended in the studies with avrPto to demonstrate a direct protein-protein interaction of the AvrPto protein with the Pto $R$ gene product (Scofield et al. 1996; Tang et al. 1996).

At least 16 genetically distinct, bacterial blight $R$ genes have been identified in cotton and numerous races of $\mathrm{Xcm}$ have been identified worldwide (Brinkerhoff 1970). As yet, none of the blight $R$ genes has been cloned, but at least 10 functional $a v r$ genes have been isolated from strains of $\mathrm{Xcm}$ (Yang et al. 1996). All are highly related and are members of the so-called avrBs3 family (Bonas et al. 1989). They are characterized by the presence of 14 to 23 tandem repeats, each 102 bp long, within the central parts of the genes. The repeat regions of the $\mathrm{Xcm}$ genes govern the avirulence specificity (Yang et al. 1994). This family of genes is present in all virulent strains of $X \mathrm{~cm}$ and is required for the induction of watersoaking during compatible infections, in addition to conferring hypersensitive necrosis in incompatible interactions (Yang et al. 1996). To determine whether the Xcm avr genes can cause specific host cell death in the absence of any other $X \mathrm{~cm}$ genes, we have carried out transient expression studies with Agrobacterium tumefaciens to introduce the genes into cotton leaves.

In order to obtain high-level expression of $\mathrm{Xcm}$ Avr proteins in plant cells, the coding regions from each of five $a v r$ genes were inserted separately into the vector pRDF9615 (Fig. 1). This plasmid contains a cauliflower mosaic virus (CaMV) 35S promoter with duplicated enhancer region (Kay et al. 1987), a $5^{\prime}$ untranslated region containing the first 70 nucleotides of tobacco mosaic virus (TMV; Goelet et al. 1982) for enhancement of translation, a unique BamHI site immediately after the initiator ATG, and the $3^{\prime}$ polyadenylation sequence from the nos gene (Bevan et al. 1983). The translational start site for the avr genes was taken as the second ATG in the open reading frames (ORFs), based on transcriptional fusions to the lacZ promoter (Yang et al. 1994) and on the presence of a strong Shine-Dalgarno sequence upstream of the second, but not the first, ATG. The presumed initiator ATG in each avr 
gene is immediately followed by a BamHI site that is in frame with the BamHI site in pRDF9615.

Each $a v r$ gene contains a second BamHI site close to the 3' end. Therefore, as an initial step in each construction, a $B a m$ HI- $B g l$ II fragment generated by polymerase chain reaction and containing the $3^{\prime}$ end of avrb6 (nucleotides 3502 to 3659; De Feyter et al. 1993) was inserted into pRDF9615, forming pRDF9652 (Fig. 1). The 3' ends of $a v r B 4$ and $a v r B 102$ are identical to that of $a v r b 6$, and the single nucleotide changes in the $3^{\prime}$ ends of $a v r b 7$ and $a v r B I n$ do not affect the avirulence specificities (Yang et al. 1994; R. de Feyter, unpublished data); therefore, the avrb6 3' end could be used in all of the constructs. The BamHI fragments containing the $5^{\prime}$ portions of each $a v r$ gene, including the central repeat regions that determine avirulence specificity, were then inserted into pRDF9652. The five resultant $a v r$ expression cassettes (e35S-avr-nos3'; Fig. 1) were excised with HindIII and inserted into the HindIII site of the binary vector pIG121-Hm (Hiei et al. 1994), and the resultant constructs mobilized into the disarmed A. tumefaciens strain AGL1 (Lazo et al. 1991). Transconjugants were grown to late exponential phase in Luria broth containing $25 \mathrm{mg}$ of kanamycin per liter. The cells were washed once, concentrated 25 -fold in sterile water, and pressure inoculated with needleless syringes into the second or third true leaves of 6- to 8-week-old cotton seedlings. Plants were maintained in a $23^{\circ} \mathrm{C}$ daytime $/ 15^{\circ} \mathrm{C}$ nighttime regime in the greenhouse.
Three to 6 days after inoculation, plants having $R$ genes responded to A. tumefaciens having the matching avr genes with brown coloration at the inoculation sites, while the susceptible control plants (Ac44) showed only a mild chlorosis and no browning (Fig. 2). Six to 14 days after inoculation, the inoculated tissue on resistant plants turned necrotic, with well-defined margins, similar in appearance to the response to avirulent $\mathrm{Xcm}$. The most rapid response occurred on cultivars 101-102B and B5-82. The responses were genotype specific, i.e., A. tumefaciens containing pRDF9987 (avrB4), pRDF9974 (avrb7), or pRDF9954 (avrBIn) induced browning and necrosis on cotton plants containing $B 4, b 7$, or BIn, respectively, but not on plants lacking the corresponding $R$ gene (Table 1). The avrB102 construct induced necrosis only on cultivars 101-102B and B5-82, consistent with its known avirulence specificity (De Feyter and Gabriel 1991). All of the avirulence constructs induced necrosis on 101$102 \mathrm{~B}$, and all except the avrb6 construct induced responses on B5-82, again consistent with the known resistance specificities of these cultivars. The only exception to this generalization was a weak and relatively slow browning induced by the $a v r B 4$ construct on all cultivars, including the susceptible variety Ac44 (Fig. 2). In conclusion, therefore, all of the avirulence constructs in A. tumefaciens induced necrosis in a plant genotype-specific manner, in the same gene-for-genes pattern as the $a v r$ genes in Xcm (De Feyter et al. 1993).

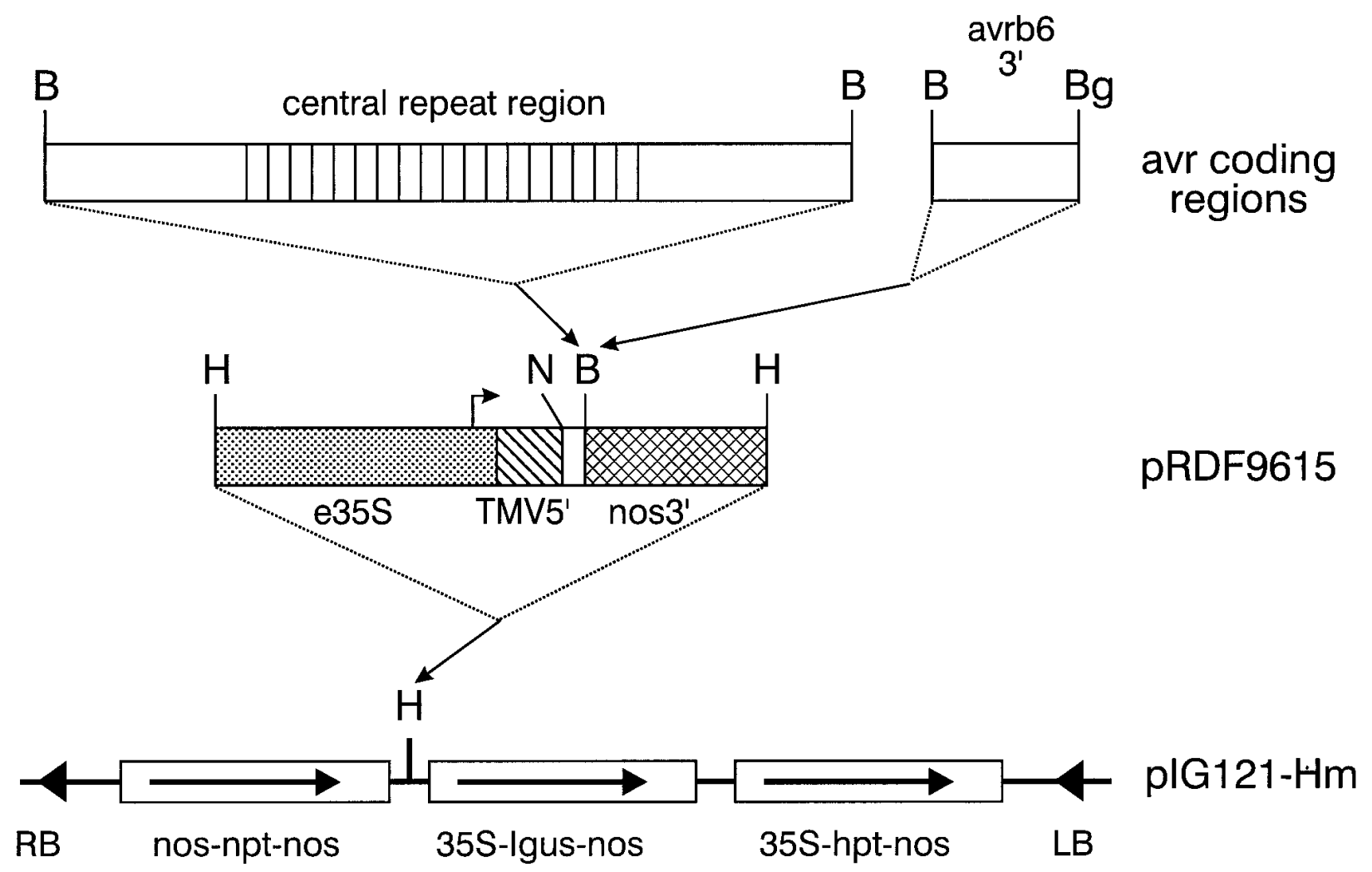

Fig. 1. Schematic diagram for the construction of avirulence (avr) genes for transient expression in cotton cells: avr open reading frames were inserted into the BamHI site of pRDF9615 as two segments, the smaller 3' region being derived from avrb6. Expression signals of pRDF9615 include an enhanced 35S promoter (e35S), the 5' untranslated region from tobacco mosaic virus (TMV5') and nopaline synthase (nos) 3' polyadenylation signal. Binary vector pIG121Hm contains a 35S-driven intron containing $\beta$-glucuronidase (gus) gene (Igus) in addition to genes conferring resistance to kanamycin (npt) and hygromycin (hpt) and T-DNA border sequences (RB, LB). Restriction site abbreviations: BamHI, B; BglII, Bg; HindIII, H; NcoI, N. 
To establish that transfer of the T-DNA from A. tumefaciens into the cotton cells was required for induction of the observed necrosis, plasmids with the $a v r$ genes but without T-DNA sequences were introduced into strain AGL1. The pUFR series of plasmids are mobilizable by conjugation between bacteria but cannot be transferred into plant cells. In these plasmids, expression of the $a v r$ genes was driven from their native promoters with the exception of $a v r B 4$, which is expressed from the strong lac $Z$ promoter. Transconjugants containing pUFR180 (lac-avrB4, avrb6), pUFR163 (avrb7), pUFR156 (avrBIn), and pUFR157 (avrB102) (De Feyter et al. 1993) were grown and used to inoculate cotton in a fashion identical to that described above. No browning or necrosis was observed, even after 18 days, indicating that expression of the avr genes in A. tumefaciens itself is insufficient to cause the observed hypersensitive responses.

To determine whether the site of action of the Avr proteins was symplastic or apoplastic, a second series of 35S-avr constructs was made by inserting the $a v r$ coding regions into the vector pRDF9574. This plasmid is identical to pRDF9615 except that it contains a 90-bp sequence encoding the 30-aminoacid signal peptide of the pea vicilin gene (Higgins et al.
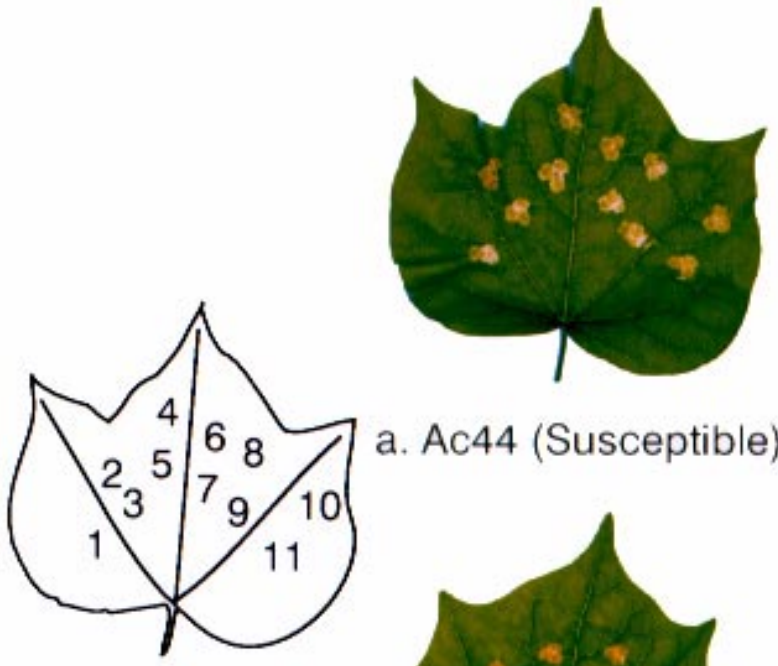

a. Ac44 (Susceptible)

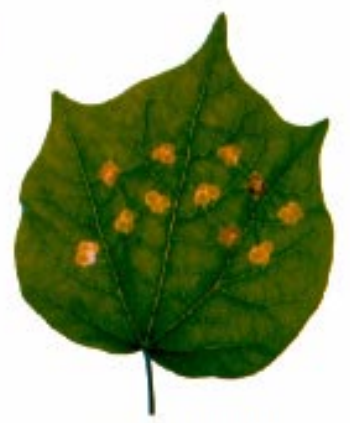

d. $B \ln (B / n)$

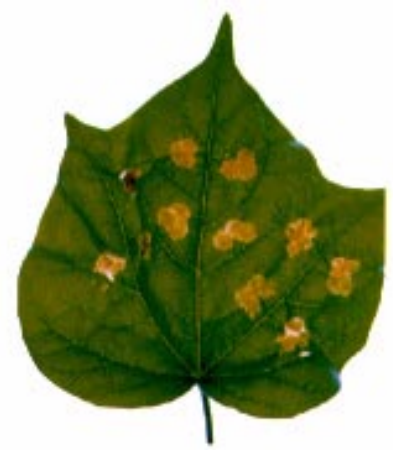

b. B4 (B4)

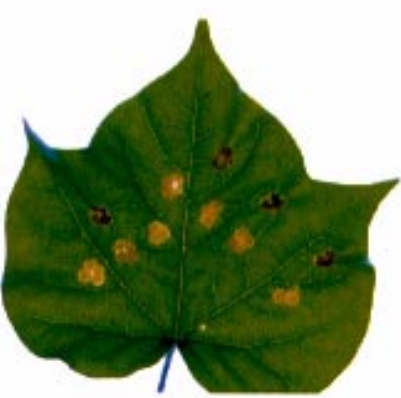

e. B5-82 (multiple)

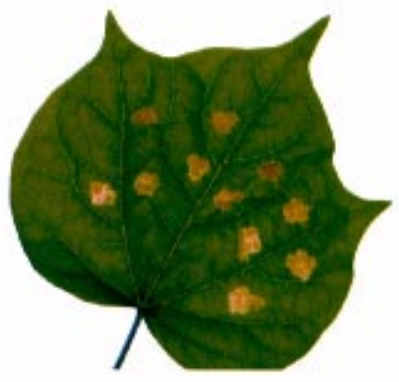

c. b7(b7)

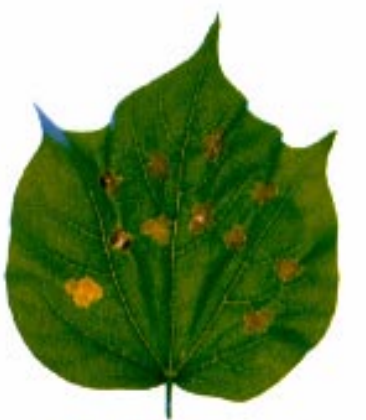

f. 101-102B (multiple)

Fig. 2. Photographs of cotton leaves 6 days after inoculation with Agrobacterium tumefaciens cultures containing the following avirulence (avr) genes within the T-DNA: 1, none (pIG121-Hm); 2, avrB4; 3, SP-avrB4; 4, avrb6; 5, SP-avrb6; 6, avrb7; 7, SP-avrb7; 8, avrBIn; 9, SP-avrBIn; 10, avrB102; 11, $S P$-avrB102. Cotton cultivars are indicated for each leaf, with known resistance $(R)$ genes in parentheses. Results are given in more detail in Table 1.

Table 1. Hypersensitive responses induced in cotton leaves, 6 days after inoculation, by transient expression of Xanthomonas campestris pv. malvacearum avirulence genes

\begin{tabular}{|c|c|c|c|c|c|c|}
\hline \multirow[b]{2}{*}{ Avirulence gene construct } & \multicolumn{6}{|c|}{ Cotton cultivar (resistance $[R]$ gene specificity) } \\
\hline & Ac44 (none) & AcB4 (B4) & $\operatorname{Acb} 7(b 7)$ & AcBIn (BIn) & B5-82 (multiple) ${ }^{\mathrm{a}}$ & 101-102 (multiple) $)^{\mathrm{b}}$ \\
\hline pIG121 (vector alone) & - & - & - & - & - & - \\
\hline pRDF9987 (avrB4) & $-^{c}$ & ++ & $-^{c}$ & $-^{\mathrm{c}}$ & +++ & ++ \\
\hline pRDF9945 (SP-avrB4) & - & + & - & - & + & ++ \\
\hline pRDF9948 (avrb6) & - & - & - & - & + & ++ \\
\hline pRDF9965 (SP-avrb6) & - & - & - & - & - & + \\
\hline pRDF9974 (avrb7) & - & - & ++ & - & +++ & ++ \\
\hline pRDF10010 (SP-avrb7) & - & - & + & - & + & ++ \\
\hline pRDF9954 (avrBIn) & - & - & - & ++ & +++ & ++ \\
\hline pRDF10000 (SP-avrBIn) & - & - & - & + & + & ++ \\
\hline pRDF9998 (avrB102) & - & - & - & - & +++ & ++ \\
\hline pRDF10251 (SP-avrB102) & - & - & - & - & + & ++ \\
\hline
\end{tabular}

a Single $R$ locus with multiple specificities.

${ }^{\mathrm{b}}$ Multiple $R$ loci with multiple specificities (Brinkerhoff 1970). SP, presence of signal peptide sequence;,,$++++++=$ hypersensitive responses, decreasing in intensity of response; -, absence of a hypersensitive response.

c A weak, nonspecific response to avrB4 observed in all cultivars including Ac44. 
1988), modified to remove an internal HindIII site, between the initiator ATG codon and the BamHI cloning site. Expression of this series of constructs (35S-SPavr-nos3') in plant cells would thus result in Avr proteins with $\mathrm{NH}_{2}$-terminal signal peptides that would, with correct processing, direct the synthesized Avr proteins to the apoplast. It has been shown that addition of small $\mathrm{NH}_{2}$-terminal sequences has no effect on avirulence activity or specificity of Avr proteins in $\mathrm{Xcm}$ (Yang et al. 1994).

The five 35S-SPavr-nos3' cassettes were introduced into pIG121-Hm and the resultant constructs mobilized into AGL1 and used to inoculate cotton as before. Genotype-specific browning and necrosis were observed on cotton cultivars as with the cytoplasmic constructs, but in every case the hypersensitive responses were weaker and developed more slowly for the apoplastic constructs. This was most marked for cultivar B5-82 (Fig. 2).

Both sets of 35S-avr constructs also contained a 35S-driven $\beta$-glucuronidase (gus) gene, present on the binary vector pIG121-Hm, that contains an intron to prevent bacterial expression of GUS activity. Cotton leaves were stained for GUS activity 3 days after inoculation with A. tumefaciens. Strong GUS expression was observed at inoculation sites on leaves of all cultivars except for 101-102B. However, leaves of this cultivar inoculated with AGL1(pIG121-Hm) did produce GUS activity, showing that 101-102B leaves are capable of expressing the intron-gus gene. These results show that protein expression was occurring prior to induction of necrosis in all cotton/avr interactions except for those on 101-102B, which reacts more quickly than any other cultivar to $a v r$ gene expression.

Based on the presence of nuclear localization signals (NLS) within the Xcm avr genes, Yang and Gabriel (1995) suggested that the Avr proteins function internally in cotton cells to cause hypersensitive cell death. NLS sequences are also present and function in avrBs3 (Van den Ackerveken et al. 1996). The presence of the NLS sequences suggests that the Avr proteins are imported into nuclei for their avirulence activity. Our data showing that addition of a signal peptide sequence to the Avr proteins reduced the avirulence activity is consistent with this model, although it is also possible that the stability of the proteins was affected if signal peptide cleavage did not occur. It is clear, however, that expression of the $\mathrm{Xcm}$ Avr proteins in cotton cells led to the observed hypersensitive cell death. Importantly, the reactions were genotype specific, requiring the presence of corresponding $a v r$ and $R$ genes. These results also lead to the possibility of isolation of the $R$ genes from cotton by direct interaction with Avr proteins.

\section{ACKNOWLEDGMENTS}

We thank Roger Mummery for excellent technical assistance with this work and Danny Llewellyn for helpful discussions. We acknowledge the support of the Cotton Research and Development Corporation (grant CSP84C).

\section{LITERATURE CITED}

Bent, A. F. 1996. Plant disease resistance genes: Function meets structure. Plant Cell 8:1757-1771.

Bevan, M., Barnes, W. M., and Chilton, M. D. 1983. Structure and transcription of the nopaline synthase gene region of T-DNA. Nucleic Acids Res. 11:369-385.

Bonas, U., Stall, R. E., and Staskawicz, B. 1989. Genetic and structural characterization of the avirulence gene avrBs3 from Xanthomonas campestris pv. vesicatoria. Mol. Gen. Genet. 218:127-136.

Brinkerhoff, L. A. 1970. Variation in Xanthomonas malvacearum and its relation to control. Annu. Rev. Phytopathol. 8:85-110.

De Feyter, R., and Gabriel, D. W. 1991. At least six avirulence genes are clustered on a 90-kilobase plasmid in Xanthomonas campestris pv. malvacearum. Mol. Plant-Microbe Interact. 4:423-432.

De Feyter, R., Yang, Y., and Gabriel, D. W. 1993. Gene-for-genes interactions between cotton $R$ genes and Xanthomonas campestris pv. malvacearum avr genes. Mol. Plant-Microbe Interact. 6:225-237.

Goelet, P., Lomonosoff, G. P., Butler, P. J. G., Akam, M. E., Gait, M. J., and Karn, J. 1982. Nucleotide sequence of tobacco mosaic virus RNA. Proc. Natl. Acad. Sci. USA 79:5818-5822.

Gopalan, S., Bauer, D. W., Alfano, J. A., Loniello, A. O., He, S. Y., and Collmer, A. 1996. Expression of the Pseudomonas syringae avirulence protein AvrB in plant cells alleviates its dependence on the hypersensitive response and pathogenicity (Hrp) secretion system in eliciting genotype-specific hypersensitive cell death. Plant Cell 8: 1095-1105.

Hiei, Y., Ohta, S., Komari, T., and Kumashiro, T. 1994. Efficient transformation of rice (Oryza sativa L.) mediated by Agrobacterium and sequence analysis of the boundaries of the T-DNA. Plant J. 6:271-282.

Higgins, T. J. V., Newbigin, E. J., Spencer, D., Llewellyn, D. J., and Craig, S. 1988. The sequence of a pea vicilin gene and its expression in transgenic tobacco plants. Plant Mol. Biol. 11:683-695.

Kay, R., Chan, A., Daly, M., and McPherson, J. 1987. Duplication of CaMV 35S promoter sequences creates a strong enhancer for plant genes. Science 236:1299-1302.

Keen, N. T. 1990. Gene-for-gene complementarity in plant-pathogen interactions. Annu. Rev. Genet. 24:447-463.

Lazo, G. R., Stein, P. A., and Ludwig, R. A. 1991. A DNA transformation-competent Arabidopsis genomic library in Agrobacterium. Biotechnology 9:963-967.

Leach, J. E., and White, F. F. 1996. Bacterial avirulence genes. Annu. Rev. Phytopathol. 34:153-179.

Scofield, S. R., Tobias, C. M., Rathgen, J. P., Chang, J. H., Lavelle, D. T., Michelmore, R. W., and Staskawicz, B. J. 1996. Molecular basis of gene-for-gene specificity in bacterial speck disease of tomato. Science 274:2063-2065.

Tang, X., Frederick, R. D., Zhou, J., Halterman, D. A., Jia, Y., and Martin, G. B. 1996. Initiation of plant disease resistance by physical interaction of AvrPto and Pto kinase. Science 274:2060-2063.

Van den Ackerveken, G., Marois, E., and Bonas, U. 1996. Recognition of the bacterial avirulence protein AvrBs3 occurs inside the plant cell. Cell 87:1307-1316.

Yang, Y., De Feyter, R., and Gabriel, D. W. 1994. Host-specific symptoms and increased release of Xanthomonas citri and X. campestris pv. malvacearum from leaves are determined by the 102-bp tandem repeats of pthA and $a v r b 6$, respectively. Mol. Plant-Microbe Interact. 7:345-355

Yang, Y., and Gabriel, D. W. 1995. Xanthomonas avirulence/pathogenicity gene family encodes functional plant nuclear targeting signals. Mol. Plant-Microbe Interact. 8:627-631.

Yang, Y., Yuan, Q., and Gabriel, D. W. 1996. Watersoaking function(s) of XcmH1005 are redundantly encoded by members of the Xanthomonas avr/pth gene family. Mol. Plant-Microbe Interact. 9:105-113. 